IUCAA - 11/96

March 96

\title{
Classification of Galactic Collisions
}

\section{By}

K.S.V.S. Narasimhan, K.S. Sastry and Saleh Mohammed Alladin Astronomy and Astrophysics 


\title{
CLASSIFICATION OF GALACTIC COLLISIONS
}

K.S.V.S.Narasimhan, K.S.Sastry ${ }^{\star}$ and Saleh Mohammed Alladin

IUCAA, Ganeshkhind, Pune 411007

\begin{abstract}
We propose a classification scheme for galactic collisions based on the fractional changes in the binding energy and the orbital energy. We give examples of actual observed pairs of interacting galaxies for the various types of collision proposed in the scheme.
\end{abstract}

\section{Introduction}

Though originaly thought to be exciting but rare phenomenon, galaxy collisions have, over the years, been considered to play an important role in the formation and evolution of galaxies. In galactic collisions translational energy, E, of galaxies is generally transferred to the energy, $\mathrm{U}$, of constituent stars. In this paper we propose a classification scheme for galactic collisions based on this energy transfer and give examples of the various types from observed pairs of interacting galaxies.

\section{Earlier Work}

Zwicky (2959) proposed a classification of temporary multiple galaxies based on the increasing order of the intensity of tidal effects resulting from galactic encounters, as follows:

\footnotetext{
*Permanent address : Department of Astronomy, Osmania university, Hyderabad - 500007 .
} 
(i) Galaxies which may encounter each other without any significant effect on each other, with only an insignificant rearrangement within them; (ii) galaxies which may encounter each other at very close range resulting in the loss of energy from the orbital motion (translational energy) of the galaxies and corresponding gain in the internal degrees of freedom and (iii) galaxies which may collide with each other head-on or nearly head-on resulting in considerable disruption of the galaxies or even tidal capture leading to merger.

Heggie (1975) has classified encounters between a binary star and a single star. He has summarised the results of the encounters in a classification scheme based on changes in energy, which in our notation is as follows:

Case (i) : $\frac{\Delta U}{|U|}<0 \quad$ : - $\begin{aligned} & \text { De-excitation of the } \\ & \text { binary }\end{aligned}$ Case (ii) : $0<\frac{\Delta U}{|u|}<1 ; \frac{|\Delta E|}{E}<1$ : Excitation of the binary Case (iii): $0<\frac{\Delta U}{|U|}<1 ; \frac{|\Delta E|}{E} \geqslant 1:$ Resonance, single star Case (iv): $\frac{\Delta U}{|U|} \geqslant 1 ; \frac{|\Delta E|}{E}<1: \begin{aligned} & \text { Ionisation, binary } \\ & \text { disrupts }\end{aligned}$ Case (v): $\frac{\Delta U}{|U|} \geqslant 1 ; \frac{|\Delta E|}{E} \geqslant 1:$ Exchange Here $\frac{\Delta U}{|U|}$ and $\frac{|\Delta E|}{E}$ are fractional changes in the binding energy of the binary and the total translational energy of the system. 
A quantitative classification based on Zwicky's qualitative classification was given by Sastry and Alladin (1977, 1979) who classified galactic collisions according to increasing intensity of tidal effects into types $A, B, C$ and $D$ as follows:

Type A : Change in size and mass of the galaxy both negligible

$$
\left(\frac{\Delta R}{R}<0.1 ; \frac{\Delta M}{M}<0.01 ; \frac{\Delta U}{|U|}<1\right)
$$

Type B : Significant change in size or in mass or in both

$$
\text { ( } \left.\frac{\Delta R}{R} \geqslant 0.1 \text { and /or } \frac{\Delta M}{M} \geqslant 0.01 ; \frac{\Delta U}{U 1}<1\right)
$$

Type $C$ : Test galaxy captured by the field galaxy

$$
\left(\frac{|\Delta E|}{E} \geqslant 1\right)
$$

Type $D$ : Test galaxy disrupted by the field galaxy

$$
\left(\frac{\Delta U}{\| u} \geqslant 1\right)
$$

Sastry and Alladin's classification was used by Miller (1983) in his study of galaxy clusters.

\section{Present Classification Scheme}

In this paper we present a classification scheme of galaxy collisions based entirely on energy changes which is analogous to that proposed by Heggie for stellar encounters but with minor modification. Case (i) rarely happens in galactic encounters. It occurs under very special conditions (Byrdet al 1986). We modify cases (i) and (ii) in Heggie to

Case (i): $\frac{\Delta U}{|U|}<0.1 ; \frac{|\Delta E|}{E}<1:$ A type collision: Tidal effects small

Case (ii): $0.1 \leqslant \frac{\Delta U}{|U|}<1 ; \frac{|\Delta E|}{E}<1:$ B type collision: Tidal effects moderate 
Many interesting features such as bridges, tails and rings are formed in B type collisions. Cases (iii), (iv) and (v) remain the same as in Heggie. These correspond to capture without disruption (C type), disruption without capture (D type) and disruption with capture (cD type) in the case of two galaxies.

This classification scheme is simpler than that earlier proposed by Sastry and Alladin (1977). The changes in mass and dimension are simply related to $\Delta U$ as indicated by Dekel et al (1980). Hence it is not necessary to include them as additional parameters.

\section{Equations For Energy Changes}

We give the basic equations used in the present classification scheme, for estimating energy changes. These equations have been derived under impulsive approximation (Spitzer, 1958) using polytropic or Plummer model for galaxies. Impulsive approximation gives reasonable estimates even for low velocity, highly penetrating collisions (Aguilar and white, $1985)$.

For a perturber o mass $M$ moring in a relative orbit of

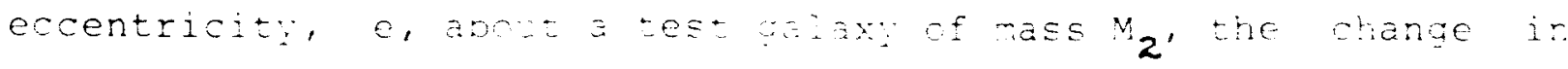
the internai enery, $\Delta U_{2}$, of the test galaxy is given by thladin and Narasimian, 198:; Narasimbar ara Alladin, 1993): 


$$
\Delta U_{2}=\left[\frac{\pi}{1+e}\right]^{2} \frac{G^{2} M_{1}^{2} M_{2}}{p^{4} V_{p}^{2}}\left(R_{\text {rms 2 }}\right)^{2} ; e \leqslant 1
$$

and

$$
\begin{gathered}
\Delta U_{2}=\frac{1}{2} \frac{G M_{1}^{2} M_{2}}{p^{3}\left(M_{1}+M_{2}\right)}\left\langle e_{i}^{2}\right\rangle\left(R_{\text {rms z }}\right)^{2} ; e>1 \\
\text { where } v p=\frac{G\left(M_{1}+M_{2}\right)}{p}(1+e)
\end{gathered}
$$

Vp being the velocity at the minimum separation $p$ of the galaxies and Rims is the root mean square radius of the galaxy. The fractional change in the binding energy, $\Delta U_{2} /\left|U_{2}\right|$ is given by $\frac{\Delta U_{2}}{\left|U_{2}\right|}=\frac{2}{\beta_{n}} \frac{\pi^{2}}{(1+e)^{3}} \frac{M_{12}^{2}}{1+M_{12}}\left(\frac{R_{\text {rms 2 }}}{p}\right)^{3} ; e \leqslant 1$

and

$$
\frac{\Delta U_{2}}{\left|U_{2}\right|}=\frac{\left\langle e_{i}^{2}\right\rangle}{\beta_{n}} \frac{M_{12}^{2}}{1+M_{12}}\left(\frac{R_{\text {rms }}}{p}\right)^{3} ; \quad e>1
$$

Where $\beta_{n}$ varies from 0.5 to 0.4 in a polytropic model as polytropic index $\mathrm{n}$ increases from 0 top 4 and $\mathrm{M}_{12}=\mathrm{M}_{1} / \mathrm{M}_{2}$.

The change in the orital energy is obtained from

$$
\Delta E=-\left(\Delta U_{1}+\Delta U_{2}\right)
$$

In a hyperbolic encounter, the criterion for capture is $\frac{|\Delta E|}{E} \geqslant 1$ or $E_{f}<0$ where $E_{f}$, the final orbital energy at the end of the encounter, has been obtained from:

$$
E_{f}=\frac{1}{2} \frac{G M_{1} M_{2}}{M_{1}+M_{2}} V_{p}^{2}+\frac{G M_{1} M_{2}}{p}-\frac{\left(\Delta u_{1}+\Delta U_{2}\right)}{2}
$$

For Plumber model galaxies of unequal mass, we have for a head-on collision (Ahmed 1979).

5 


$$
\begin{aligned}
& \frac{\Delta U_{2}}{\left|U_{2}\right|}=\frac{64}{3 \pi} \frac{G M_{1}^{2} \alpha_{2}}{M_{2} V_{p}^{2} \alpha_{1}^{2}} B\left(\alpha_{12}\right) ; \alpha_{12}=\alpha_{1} / \alpha_{2} \\
& \frac{|\Delta E|}{E}=\frac{\alpha_{12}^{2}+M_{12}}{2\left(1+M_{12}\right)} \frac{B}{A^{2}}\left(\frac{V_{e s c}}{V_{p}}\right)^{4}
\end{aligned}
$$

Where $\alpha$ is scale length and vesc is the escape velocity of the galaxies. $A$ and $B$ are functions of $\alpha_{12}$. Namboodiri et al (1987) define a dimensionless parameter $\mathcal{V}$ given by

$$
\nu=\frac{\pi^{2}}{(1+e)^{3}} \frac{M_{12}^{2}}{1+M_{12}}\left(\frac{R_{2}}{p}\right)^{3} ; e \leqslant 1, p \approx\left(R_{1}+R_{2}\right)
$$

They find that bridges and tails are formed in the disk galaxy when $0.1 \leq \nu \leq 0.7$

The fractional change in the binding energy of a disk galaxy due to a head-on collision with a spherical galaxy ( $S$ ) is given by (Chatterjee 1984)

$$
\frac{\Delta U}{|U|}=\frac{G M_{S}^{2} R_{D}}{V^{2} M_{D} R_{S}^{2}} \beta_{D}
$$

Where $\beta_{\mathcal{D}}$ is a function of the galaxy models, the ratio of radii $\mathrm{RS} / \mathrm{RD}$ and the inclination of the disk to the relative orbital motion of the galaxies. Chatterjee finds that ring galaxies are formed when $0.4 \leq \frac{\Delta U}{|U|} \leq 1.8$ and $\frac{\Delta U}{|U|} \geq 2$ implies complete disruption.

\section{Classification of some Observed Pairs of Galaxies}

In this section we classify pairs of interacting galaxies according to the scheme given in cection 3 using the observed data and the equations given in section 4 . 
(i) NGC $1587-1588(=\mathrm{K} 99)$ is a pair of ellipticai galaxies showing off-centering of inner versus outer isophotes (Bonfanti et al 1995), U-shaped velocity profiles and an enhancement in the velocity dispersion between the galaxies (Combes et al 1995). All these are attributed to tidal interaction.

From the simulations of Borne (1988), we adopt $M_{1}=1.95 x$ $10^{11} \mathrm{M}_{\Theta^{\prime}} \mathrm{M}_{2}=0.65 \times 10^{11} \mathrm{M}_{\odot}$. We take $\mathrm{Vp} \approx 390 \mathrm{~km} / \mathrm{sec}$, allowing for projection effects, $p=20 \mathrm{kpc}$. Fish's law (Alladin et al 1975 ) yields $R_{1}=14 \mathrm{kpc}, \mathrm{R}_{2}=8 \mathrm{kpc}, \mathrm{Rrms}_{1}=2.8 \mathrm{kpc}$ and $\mathrm{Rrms}_{2}=$ $1.6 \mathrm{kpc}$. This gives $\mathrm{e}=1.25$ and $\frac{\Delta U_{2}}{\left|U_{2}\right|}=0.03$ and $E_{f}>0$ implying that the orbit remains hyperbolic after the collision. We designate this collision as of Type A.

(ii) NGC 2672-2673(=K175=Arp 167) is again another pair of isolated elliptical galaxies showing similar characteristics of interaction. We use the collision parameters as given in Balcells et al (1989). With $\mathrm{M}_{1}=4 \times 10^{\prime 1} \mathrm{M}_{\odot} \mathrm{M}_{2}=4 \times 10^{\prime 0} \mathrm{M}_{\odot^{\prime}}$ $R_{1}=20 \mathrm{kpc}, R_{2}=6.3 \mathrm{kpc}, \mathrm{Rrms}_{1}=4 \mathrm{kpc}$, Rrms $_{2}=1.26 \mathrm{kpc}, \mathrm{Vp}=$ $540 \mathrm{~km} / \mathrm{sec}$ and $\mathrm{p}=16 \mathrm{kpc}$, we obtain $e=1.1, \frac{\Delta U_{2}}{\left|U_{2}\right|}=0.01$ and $E_{f}>0$. This is also a collision of Type

(iii) NGC $4782-4783(36278)$ is a lose pair of interacting galaxies. Madejsky and Bien (1993) have presented a high velocity and deeply penetrating hyperbolic encounter modei which agrees with the observed data. 
Following Borne et al (1988), we chose $M_{1}=8.1 \times 10^{11} \mathrm{M}^{\prime} M_{2}$ $=5.6 \times 10^{\prime \prime} \mathrm{M}_{0}$ and using Fish's law we obtain Rrms $=6 \mathrm{kpc}$ and Rrms $_{2}=5 \mathrm{kpc}$. Taking $V p=960 \mathrm{~km} / \mathrm{sec}$ and $\mathrm{p}=17 \mathrm{kpc}$ as in Madejsky and Bien (1993), we get $\frac{\Delta U_{2}}{\left|U_{2}\right|}=0.1$ indicating that the collision is of Type B.

(iv) NGC $5194-5195(=M 51=$ VVI $)$ is the Whirlpool nebula. From Toomre's (1972) model, we adopt $M_{1}=13.5 \times 10^{10} M_{0} M_{2}=4.5$ $\times 10^{10} \mathrm{M}_{\odot^{\prime}} \mathrm{p}=25 \mathrm{kpc}$ and $\mathrm{e}=0.8$. Taking $\mathrm{R}_{1}=15 \mathrm{kpc}, \mathrm{R}_{2}=10$ kpc (typical values for radii), we find $\nu=0.25, \frac{\Delta U_{2}}{\left|U_{2}\right|}=0.15$. $e=0.8$ implies that a capture has already taken place. The collision is of Type $C$.

(v) $\quad$ A0035 (=NGC 4650 A $=$ AM $064-0741)$

A0035 is known as Cartwheel galaxy. The collision parameters are given in Chatterjee (1984). He finds that $0.8 \leq \frac{\Delta}{T}$ $\leq 1.8$. We classify this collision as of Type D.

(vi) Arp 141 (=VV 123) pair: The peculiar nature of this interacting pair was attributed by Burbidge and Burbidge (1959) to the discuption of a spiral galaxy by an elliptical galaxy ir. a close encounter. Following Sandage (1963), we take $\mathrm{R}_{\boldsymbol{2}}=8 \mathrm{kpc}$ and $p=4.6 \mathrm{kpc}$. We assume for the elliptical galaxy a typical mass $M_{1}=3 \times 10^{12} M_{0}$. Fish's law then gives $R_{1}=55 \mathrm{kpc}, M_{2}=8$ $x \quad 10^{10} M_{\odot}$. In the absence of information about the relative velocity of the pair, we assume crudely the differential velocity 
of $3000 \mathrm{~km} / \mathrm{sec}$ of the system as $\mathrm{Vp}$. These collision parameters give $e=1.68$ and $\frac{\Delta U_{2}}{\left|U_{2}\right|} \approx 3$. This means that the spiral is getting disrupted in a hyperbolic encounter with the elliptical. We classify the collision as of Type D. Alternatively, since $p<<R_{1}$, we may regard the collision as nearly head-on and from the treatment given in Ahmed (1979) we get $\frac{\Delta U_{2}}{\left|U_{2}\right|} \approx 1.5$ and $\frac{|\Delta E|}{E} \ll 1$. Hence in this case also the collision is of type $D$. We note also that Chatterjee's (1984) criterion for the formation of ring galaxies is satisfied. This may possibly explain the ring structure observed in the pair.

(vii) NGC 2444-2445 (=Arp $143=$ VV 117) is an interacting pair of elliptical (E4) and spiral (SC) galaxies. Following Narasimhan and Alladin (1985), we adopt $M_{1}=1.4 \times 10^{11} M_{0}, M_{2}=9$ $\times 10^{10} \mathrm{M}_{\sigma^{\prime}} \mathrm{e}=0.3, \mathrm{p}=12 \mathrm{kpc}$ and representing the galaxies by polytropes of indices $n_{1}=4$ and $n_{2}=3$ respectively, we get $\frac{\Delta U_{2}}{|\Delta E|}$ $=1.6$ and $\frac{|\Delta E|}{E}=1.8$. Thus $\frac{\Delta U}{|U|}>1, \frac{|\Delta E|}{E}>1$. We classify this collision as as Type CD. The spiral will merge with the elliptical with considerable disruption.

\section{Conclusion}

We have classified galaxies in increasing order of tidal effects; $A$ : small tidal effect; $B$ : moderate tidal effect; $C$ : capture without disruption; D: disruption without capture and $C D$ : both capture and disruption. The tidal effects are estimated 
from energy changes. At least one example of each collision type is given.

\section{Acknowledgement}

We thank Professor J.V. Narlikar, Director, IUCAA, Pune for his encouragement and for providing facilities for working at IUCAA. SMA also thanks UGC, New Delhi for financial support for this project.

\section{References}

Aguilar, L.A., and White, S.D.M.: 1985, Astrophys. J. $\underline{245}, 374$. Ahmed, F.: 1979, Astrophys.Sp.Sci. 60, 293.

Alladin, S.M. and Narasimhan, K.S.V.S.: 1982, Phys.Rep. 92, 339. Alladin, S.M., Potdar, A., and Sastry, K.S.: 1975, IAU Symp. 69. 167.

Balcells, M., Borne, K.D., and Hoessel, J.G.: 1989, Astrophys. J. $\underline{236}, 655$.

Bonfanti, P.R., Rampazzo, R., Combes, F., Prugniel, P. and Sulentic, J.W.: 1995, Astron. Astrophys. 297, 28.

Borne, K.D.: 1988, Astrophys. J. $\underline{330}, 61$.

Borne, K.D., Balcells, M. and Hoessel, J.G.: 1988, Astrophys.J. 333,567 .

Burbidge E.M. and Burbidge, G.R.: 1959, Astrophys.J. 130, 12. Byrd, G.C., Saarinen, S., and Valtonen, M.J.: 1986a, Mon.Not. R.Astr.Soc. 220,619 .

Chatterjee, T.K.: 1984, Astrophys.sp.sci. $106,309$. 
Combes, F., Rampazzo, R., Bonfanti, P.R., Prugniel, P. and Sulentic, J.W.: 1995, Astron. Astrophys, 297, 37.

Dekel, A., Lecar, M; and Shaham, J.: 1980, Astrophys. J. 241, 946.

Heggie, D.C.: 1975, Mon.Not.R.Astr.Soc. 173, 729.

Madejsky, R., and Bien, R.: 1993, Astron. Astrophys. 220, 383 .

Miller, G.E.: 1983, Astrophys. J. 268, 495.

Namboodiri, P.M.S., Kochhar, R.K.; and Alladin, S.M.: 1987, Bull. Astron. Soc. India, 15, 186 .

Narasimhan, K.S.V.S, and Alladin, S.M.: 1983, Bull. Astron Soc. India, 11, 221 .

Narasimhan, K.S.V.S, and Alladin, S.M.: 1985, Bull. Astro Soc. India, 13,395 .

Sandage, A.: 1963, Astrophys. J. 183, 863.

Sastry, K.S. and Alladin, S.M.: 1977, Astrophys. Sp. Sci, 46, 285 .

Sastry, K.S. and Alladin, S.M.: 1979, Astrophys. Sp.Sci, 63, 203. Spitzer, L.: 1958, Astrophys. J. 127, 17 .

Toomre, A. and Toomre, J.: 1972, Astrophys, J. 178, 623.

Zwicky, F.: 1959, Hand buch der Physik, 53, (Springer-verlag), p. 374. 
